\title{
PRIMEIRO REGISTRO DE GALHAS EM PANÍCULAS DE CAJAZEIRA CAUSADAS POR Clinodiplosis (DIPTERA: Cecidomyiidae ) E AVALIAÇÃO DE GENÓTIPOS NO ESTADO DO PIAUII ${ }^{1}$
}

\author{
JAQUELINE ZANON DE MOURA², STAINI ALVES BORGES 3 , \\ PAULO ROBERTO RAMALHO SILVA ${ }^{4}$, VALÉRIA CID MAIA ${ }^{5}$, TAILINE ISABEL ALVES BORGES ${ }^{6}$
}

RESUMO-A cajazeira (Spondias mombin L.) é uma Anacardiaceae que se destaca pela considerável importância social e econômica no Nordeste brasileiro. Vários autores mencionam insetos que atacam folhas, ramos e frutos de cajazeiras; no entanto, em relação às flores, as informações são limitadas. Cecidomyiidae é uma família bastante diversificada de Nematocera (Diptera). Cecidomyiinae têm hábitos variados e grande riqueza de espécies fitófagas. Neste trabalho, objetivou-se a identificação de uma espécie de Cecidimyiinae galhador em cajazeira, caracterização dos sintomas do ataque e avaliação do nível de infestação em panículas da cajazeira em diferentes genótipos. O experimento foi desenvolvido em pomar de cajazeira do Departamento de Fitotecnia do Centro de Ciências Agrárias da Universidade Federal do Piauí. Foram avaliados oito genótipos: JL 15, LA1, Fazenda Soares I, Fazenda Soares II, Fazenda Soares III, Major, Vagner e Jardim. O inseto foi identificado como Clinodiplosis sp. Considerando-se a alta especificidade de Cecidomyiinae (Clinodiplosini) na relação galhador X planta hospedeira, a espécie de Clinodiplosis associada a Spondias mombin provavelmente é nova. Os genótipos Major, LA 1, Soares I e Jardim apresentaram menor infestação. Já os genótipos JL 15, Vagner, Soares III e Soares II apresentaram maior infestação. Pode-se concluir que o nível de infestação das cajazeiras por Clinodiplosis sp. varia de acordo com o genótipo avaliado.

Termos para indexação: Spondias mombin L., Anacardiaceae, Inseto-praga.

\section{FIRST RECORD OF GALLS IN HOG PLUM (Spondias mombim L.) PANICLES CAUSED BY Clinodiplosis (Diptera: Cecidomyiidae) AND EVALUATION OF GENOTYPES IN THE STATE OF PIAUÍ}

\begin{abstract}
Hog plum (Spondias mombim L.) is an Anacardiaceae of considerable social and economical importance in Brazilian northeast. Various authors mentioned insects that affect leaves, branches and the fruits, but information related to flower is limited. Cecidomyiidae is a very well diversified family of Nematocera (Diptera). Cecidomyinae has varied habits and a great variety of phytophagaus species. This study had as objective the identification of a galling Cecidomyiinae species, characterization of the attack symptoms and assesses the level of infestation of this species in panicles of different hog plum genotypes. The experiment was developed in a hog plum orchard from the Departamento de Fitotecnia from Universidade Federal do Piauí. Eight genotypes were evaluated: JL 15, LA 1, Fazenda Soares I, Fazenda Soares II, Fazenda Soares III, Major, Vagner and Jardim. The insect was identified as Clinodiplosis sp.. Considering the high specificity of galling Cecidomyiinae (Clinodiplosini) with respect to host plants, the Clinodiplosis species associated with Spondias mombim is probably new. The genotypes Major, LA 1, Soares I and Jardim showed lower infestation. The genotypes JL 15, Vagner, Soares III and Soares II showed the highest infestation. These results show that the level of infestation by Clinodiplosis sp varies with the hog plum genotype assessed.

Index terms: Spondias mombin L., Anacardiaceae, Insect pest.
\end{abstract}

\footnotetext{
${ }^{1}$ Trabalho 052-09). Recebido em: 25-02-2009. Aceito para publicação em: 08-04-2010. Parte do Trabalho de Conclusão de Curso do $2^{\circ}$ autor.

${ }^{2}$ Prof ${ }^{\text {a. }}$ Assistente do Dept ${ }^{\circ}$. de Engenharia da Universidade Federal do Piauí (UFPI), Campus Professora Cinobelina Elvas/Bom Jesus, BR 135 Km 03, Planalto Horizonte, Cep 64900-000, Bom Jesus - PI. E-mail: jaqueline.zanon@hotmail.com (autor correspondente). ${ }^{3}$ Engenheiro Agrônomo. E-mail: stainiborges@hotmail.com

${ }^{4}$ Prof. Associado do Dept ${ }^{\circ}$. de Fitotecnia do Centro de Ciências Agrárias da UFPI. E-mail: pramalhoufpi@yahoo.com.br ${ }^{5}$ Prof. Adjunta do Dept ${ }^{\circ}$. de Entomologia do Museu Nacional do Rio de Janeiro-Universidade Federal do Rio de Janeiro (UFRJ). Email: maiavcid@acd.ufrj.br.

${ }^{6}$ Licenciada em Biologia. E-mail: tailineisabel@hotmail.com.
} 
A cajazeira (Spondias mombin L.) é uma árvore frutífera da família Anacardiaceae, considerada cosmopolita. Vegeta espontaneamente na América Tropical e ao sul da zona equatorial (SOUZA, 1998). Encontra condições favoráveis de sobrevivência em vários ecossistemas brasileiros, notadamente no Norte e Nordeste (BOSCO et al., 2000). Possui considerável importância social e econômica. No Nordeste, é largamente explorada através do extrativismo e com grande potencial de exploração agroindustrial (SOUZA, 1998). Os frutos são utilizados na confecção de polpas, sucos, picolés, sorvetes, néctares e geleias de excelente qualidade e valor comercial. A madeira é utilizada em marcenarias, e a casca, os ramos, as folhas e as flores possuem propriedades medicinais. A cajazeira ainda não é cultivada em escala comercial, sendo considerada planta em domesticação e de exploração extrativa (SACRAMENTO; SOUZA, 2000).

Vários autores mencionam insetos, como tripes (Thysanoptera) (HICKEL, 2002), cochonilhas (Coccoidea, Hemiptera) (SANTOS, et al. 2004), lagartas (Lepidoptera), brocas (Coleoptera) e moscas (Diptera) (CARVALHO, et al. 2004), que atacam folhas, ramos e frutos de cajazeira (SACRAMENTO; SOUZA, 2000). Este artigo registra, pela primeira vez, a ocorrência de uma espécie de Cecidomyiidae (Diptera, Nematocera) atacando essa planta, e mais especificamente as flores, onde provoca a formação de galha.

Dipteras da família Cecidomyiidae são bastante diversificados, incluindo cerca de 5.451 espécies em 598 gêneros e são encontrados em todas as regiões zoogeográficas (GAGNÉ, 2004). Na região neotropical, a ocorrência de Cecidomyiidae é pouco conhecida, sendo relatado cerca de 500 espécies em 170 gêneros (MAIA, 2005). Esta família usualmente é dividida em quatro subfamílias: Catotrichinae, Lestremiinae, Porricondylinae e Cecidomyiinae. As três primeiras são pouco diversificadas e incluem essencialmente espécies fungívoras. Já Cecidomyiinae têm grande riqueza de espécies de hábitos alimentares variados; contêm formas fungívoras, mas principalmente fitófagas (galhadoras ou de vida livre) e predadoras (MAIA, 2005).

Galhas entomógenas têm uma estrutura extremamente harmônica. Suas camadas celulares dispõem-se em arranjos que formam verdadeiros tecidos, caracterizados por extensas alterações estruturais e fisiológicas, além de um crescimento anormal. Essas formas de crescimento dão origem a estruturas de tamanho e aspecto definidos, típica ação de Cecidomyiidae: esférica, esverdeada, com pelos curtos distribuídos por toda a superfície. Possuem uma única câmara onde as larvas se desenvolvem. O fim do crescimento da galha coincide com o término do crescimento larval, quando finalmente um inseto adulto emerge, deixando o pupário semienterrado na cavidade de saída. Este é um traço bastante generalizado entre os cecidomiídeos cecidógenos (FERNANDES; MARTINS, 1985).

Como a cajazeira se encontra em fase de domesticação, é importante conhecer as pragas em potencial, uma vez que podem representar um entrave à produção. Nesse contexto, a seleção de genótipos resistentes torna-se particularmente interessante.

Devido a sua importância social e econômica, fato comprovado pela crescente comercialização de seus frutos e produtos processados, a identificação desse Cecidomyiidae galhador faz-se fundamental, uma vez que ele afeta diretamente os órgãos produtivos e reprodutivos da cajazeira, inviabilizando as flores e, consequentemente, os frutos. Além de visar à identificação desse inseto, este trabalho busca também caracterizar os sintomas do ataque e avaliar o nível de infestação das panículas da cajazeira em diferentes genótipos para se detectarem possíveis fontes de resistência a essa praga em potencial.

$\mathrm{O}$ experimento foi desenvolvido em pomar de cajazeira do Departamento de Fitotecnia - DF, do Centro de Ciências Agrárias-CCA da Universidade Federal do Piauí-UFPI, situada a $05^{\circ} 05^{\prime}$ de latitude, $42^{\circ} 48^{\prime}$ de longitude, $74,4 \mathrm{~m}$ de altitude, com temperaturas variando de $22^{\circ}$ a $33,8^{\circ} \mathrm{C}$, umidade relativa do ar em torno de $69,2 \%$ e precipitação pluviométrica em torno de $1.300 \mathrm{~mm}$ (BASTOS; ANDRADE JUNIOR, 2000).

Para a coleta dos dados de infestação da praga, foram utilizados oito genótipos de cajazeiras: JL 15, LA 1, FAZENDA SOARES I, FAZENDA SOARES II, FAZENDA SOARES III, MAJOR, VAGNER e JARDIM, obtidos por enxertia, em processo de avaliação, plantados em 24 de fevereiro de 2004.

A coleta das panículas em floração foi realizada em dezembro de 2006, retirando-se uma de cada quadrante; cada panícula consistiu em uma repetição. Os pecíolos foram cortados com o auxílio de uma tesoura, as panículas foram armazenadas em sacos de papel e conduzidas ao laboratório. Em cada panícula, foram identificadas e contabilizadas as flores normais e flores com sintomas de ataque (galha), obtendo-se, posteriormente, o percentual de infestação. O delineamento estatístico utilizado foi o inteiramente casualizado, com oito tratamentos (genótipos) e quatro repetições (panículas). Os resultados obtidos foram submetidos à análise de variância, e as médias, testadas pelo teste de Tukey, a $1 \%$ de probabilidade. 
Visando à identificação do Cecidomyiidae, algumas flores atacadas foram dissecadas para a remoção da larva galhadora. As larvas assim obtidas foram conservadas em álcool a $70 \%$ e posteriormente montadas em lâminas permanentes de microscopia, seguindo-se a metodologia de Gagné (1989). A identificação foi feita com base na chave de gêneros de Gagné (1994).

O Cecidomyiidae galhador foi identificado como pertencente ao gênero Clinodiplosis (Cecidomyiinae, Clinodiplosini). Esse gênero é cosmopolita, sendo bastante diversificado tanto em número de espécies (93 descritas), como no hábito de vida (micófagos, fitófagos de vida livre, galhadores e predadores facultativos) (GAGNÉ, 2004). As espécies fitófagas (de vida livre ou galhadoras) estão associadas a 25 famílias de plantas, sendo esse o primeiro registro em Anacardiaceae. Na região neotropical, esse gênero compreende 18 espécies, 13 das quais assinaladas para o Brasil. Considerando-se a alta especificidade na relação galhador X planta hospedeira, a espécie associada a $S$. mombin provavelmente é nova.

Vários trabalhos indicam que as relações entre insetos formadores de galhas e plantas são específicas. $\mathrm{O}$ tecido da planta responde de formas diferentes produzindo características estruturais exclusivas de cada interação (SHORTHOUSE; ROHFRITSCH, 1992; ROHFRITSCH, 1992; RIBEIRO et al., 1999).
As flores de S. mombin atacadas por Clinodiplosis sp. caracterizam-se por apresentarem estruturas fisiológicas e morfológicas diferenciadas, sendo maiores que as flores não atacadas, esféricas e esverdeadas, com câmara única, características semelhantes às descritas por Fernandes e Martins (1985)

O genótipo Major apresentou menor infestação do galhador, diferindo significativamente dos genótipos JL 15, Vagner, Soares 3 e Soares 2, mantendo-se semelhante aos genótipos LA 1, Soares 1 e Jardim, com um grau de infestação baixo em comparação aos demais (TABELA 1).

Já o genótipo JL15 apresentou maior infestação de Clinodiplosis sp. (Diptera; Cecidomyiidae), diferindo de LA 1, Soares 1, Jardim e Major, sendo porém semelhante aos genótipos Vagner, Soares 3 e Soares 2. Os genótipos Vagner, Soares 3, Soares 2, LA 1 e Soares 1 apresentaram valores intermediários quanto ao parâmetro avaliado (TABELA 1).

Com base nos resultados obtidos no presente trabalho, pode-se concluir que: as galhas nas flores de S. mombin são induzidas por Clinodiplosis sp. (Diptera; Cecidomyiidae); as flores atacadas diferenciamse das sadias por serem esféricas e esverdeadas com câmara única; o nível de infestação das cajazeiras por Clinodiplosis sp. varia de acordo com o genótipo; dentre os genótipos avaliados, Major, Jardim, Soares 1 e LA 1 apresentam as menores infestações ao ataque.em genótipos de cajazeiras (Spondias monbin L.) por Clinodiplosis sp (Diptera; Cecidomyiidae) Teresina-Piauí, dezembro/2006.

TABELA 01 - Nível de infestação em genótipos de cajazeiras (Spondias monbin L.) por Clinodiplosis sp. (Diptera; Cecidomyiidae) Teresina-Piauí, dezembro/2006.

\begin{tabular}{crrl}
\hline Genótipos & Flores p/ Panícula & Flores infestadas & \% infestação \\
\hline JL 15 & 909,25 & 346,75 & $38,13 \mathrm{a}$ \\
Vagner & $1.305,25$ & 373,75 & $28,63 \mathrm{ab}$ \\
Soares 3 & $3.225,50$ & 799,00 & $24,77 \mathrm{abc}$ \\
Soares 2 & $3.014,75$ & 695,50 & $23,06 \mathrm{abc}$ \\
LA 1 & $1.714,75$ & 359,93 & $20,99 \mathrm{bcd}$ \\
Soares 1 & $2.204,00$ & 441,50 & $20,03 \mathrm{bcd}$ \\
Jardim & $1.921,00$ & 243,75 & $12,69 \mathrm{~cd}$ \\
Major & $1.609,75$ & 158,50 & $9,85 \mathrm{~d}$ \\
\hline
\end{tabular}

${ }^{1}$ Médias seguidas de mesma letra na coluna não diferem estatisticamente, segundo teste de Tukey, a 1\% de probabilidade. $\mathrm{CV}=26,53$ 


\section{REFERÊNCIAS}

BASTOS, E. A.; ANDRADE JUNIOR, A. S.de. Dados agrometeorológicos para o município de Teresina-PI (1980-1999). Teresina: Embrapa Meio Norte, 2000. 25p (Documentos, 47)

BOSCO, J.; SOARES, K. T.; AGUIAR FILHO, S. P. de; BARROS, R. V. A cultura da cajazeira. João Pessoa: EMEPA, 2000. 229p. (Série Documentos, 28).

CARVALHO, C.A.L. DE; SANTOS, W. DA S.; DANTAS, A.C.V.L.; MARQUES, .O.M.; PINTO, W. DA S. Moscas-das-frutas e parasitoides associados a frutos de cajazeiras em Presidente Tancredo Neves-Bahia. Magistra, Cruz das Almas, v.16, n.2, p.85-90, 2004

FERNANDES, G. W. A.; MARTINS, R. P. Tumores de plantas: as galhas. Ciência Hoje, Belo Horizonte, v.4, n.19, p.58-64,1985.

GAGNÉ, R. J. The gall midges of the Neotropical region. Ithaca: Cornell University Press, 1994. 352 p.

GAGNÉ, R. J. A Catalog of the Cecidomyiidae (Diptera) of the World. Memoirs of the Entomological Society of Washington, Washington, n. 25, p. $408,2004$.

GAGNÉ, R. J. The plant-feeding gall midges of North America. Ithaca: Cornell University Press, 1989. $356 \mathrm{p}$.

HICKEL, E.R. Espessura da polpa como condicionante do parasitismo de mosca-das-frutas (Diptera:Tephritidae) por Hymenoptera: braconidae. Ciência Rural, v.32, n.6, p.1005-1009, 2002. Disponível em: $<$ htpp://doc.doi.org > . DOI: $10.1590 /$ S0103-84782002000600014.
MAIA, V.C. Catálogo dos Cecidomyiidae (Diptera) do Estado do Rio de Janeiro. Biota Neotropica, Campinas, v.5, n.2, p.189-203, 2005.

RIBEIRO, J.E.; HOPKINS, M.J.G.; VICENTINI, A.; SOTHERS, C.A.; COSTA, M.A.S.; BRITO, J.M.; SOUZA, M.A.D.; MARTINS, L.H.P.; LOHMANN, L.G.; ASSUNÇÃO, P.A.C.L.; PEREIRA, E.C.; SILVA, C.F.; MESQUITA M.R.; PROCÓPIO, L.C. Flora da Reserva Ducke: guia de identificação das plantas vasculares de uma floresta de terra-firme na Amazônia Central. Manaus: INPA-DFID, 1999. 816p.

ROHFRITSCH, O. Patterns in gall development. In: SHORTHOUSE. J.D.; ROHFRITSCH, O. (Eds.) Biology of insect-induced galls. New York: Oxford University Press, 1992.

SACRAMENTO, C.K. do; SOUZA, F.X. de. Cajá (Spondias mombin L.). Jaboticabal: Funep, 2000. 42p. (Frutas Nativas, 4) .

SANTOS, W. da S.; CARVALHO, C. A.L. de; MARQUES, O.M. Registro de Neosilba zadolicha McAlpine \& Steyskal (Diptera: Lonchaeidae) em Umbu-cajá (Anacardiaceae). Neotropica Entomology, v.33, n.5, p.653-654, 2004, vol.33, n.5, p. 653-654. Doi: 10.1590/S1519-566X2004000500017.

SHORTHOUSE J.D.; ROHFRITSCH, O. Biology of insect-induced galls. New York: Oxford University Press, 1992.

SOUZA, F.X. de. Spondias agroindustriais e os seus métodos de propagação. Fortaleza: EMBRAPACNPAT:SEBRAE, 1998. 28 p. (Documentos, 27). 